\title{
NÚMEROS CROMOSÓMICOS DE ESPECIES DE MIMOSA (LEGUMINOSAE) DE PARAGUAY
}

\author{
por GUILLERMO SEIJO'
}

\begin{abstract}
Summary
In the present study the chromosome numbers of Mimosa xanthocentra subsp. subsericea var. subsericea, $M$. balansae, $M$. chacoënsis, $M$. rojasiiall with $2 n=26$ and $M$. lupinoides with $2 n=52$ are reported for the first time. The $2 n=52$ of $M$. somnians subsp. viscida var. viscida and of $M$. somnians subsp. somnians var. somnians are new and differ from the $2 n=26$ published before for $M$. somnians var. somnians of Argentina. $M$. debilis with $2 n=26$ also differs from the numbers reported previously for $M$. debilis var. debilis from Argentina with $2 n=52$. The $2 n=26$ of $M$. bimucronata var, adenocarpa coincides with the reported number for $M$. bimucronata var. bimucronata, and the number of $M$. polycarpa var. spegazzinii with $2 n=26$ is confirmed.
\end{abstract}

Key words: chromosome, Mimosa, polyploidy

Palabras clave: cromosoma, Mimosa, poliploidía

\section{Introducción}

Muchas de las especies de Mimosa L. que crecen en Paraguay son endémicas, presentan problemas taxonómicos y no han sido estudiadas cariológicamente. El propósito del presente trabajo es ampliar el conocimiento sobre los números cromosómicos de estas especies, establecer los niveles de ploidía en cada una y contribuir con esta información a la resolución de problemas taxonómicos existentes en el género.

\section{Material y métodos}

El material estudiado, su procedencia y coleccionistas se detallan en la Tabla 1. Los ejemplares de herbario están depositados en el Herbario del Instituto de Botánica del Nordeste (CTES). Los recuentos cromosómicos se realizaron a partir de células en división meiótica o

\footnotetext{
${ }^{1}$ Instituto de Botánica del Nordeste, Casilla de Correo 209, 3400 Corrientes, Argentina. Becario de Postgrado del CONICET
}

mitótica. Para los recuentos mitóticos se utilizaron meristemas radiculares de semillas germinadas en cajas de Petri, que se pretrataron con frío a $4^{\circ} \mathrm{C}$ por $12 \mathrm{~h}$ y luego en 8hidroxiquinoleína $(0.002 \mathrm{M})$ por $5 \mathrm{~h}$. Posteriormente se fijaron en etanol:ácido láctico (5:1), se colorearon siguiendo la técnica de Feulgen y se maceraron en una gota de orceína acética al 3\% antes del aplastado. Los preparados se hicieron permanentes utilizando Euparal como medio de montaje. Para los recuentos en meiosis se utilizaron botones florales fijados en etanol:ácido láctico (5:1). Las células madres del polen, previamente extraídas de los sacos polínicos, se colorearon con carmín acético al $3 \%$ y se hicieron permanentes con aceite de trementina.

\section{Resultados y discusión}

Los números cromosómicos de las especies estudiadas se detallan en la Tabla 1 y algunos de ellos se ilustran en la Fig. 1, según se indica en la misma Tabla. En este trabajo se estudiaron 17 accesiones pertenecientes a 10 taxones. 
Tabla 1. Números cromosómicos y procedencia del material estudiado

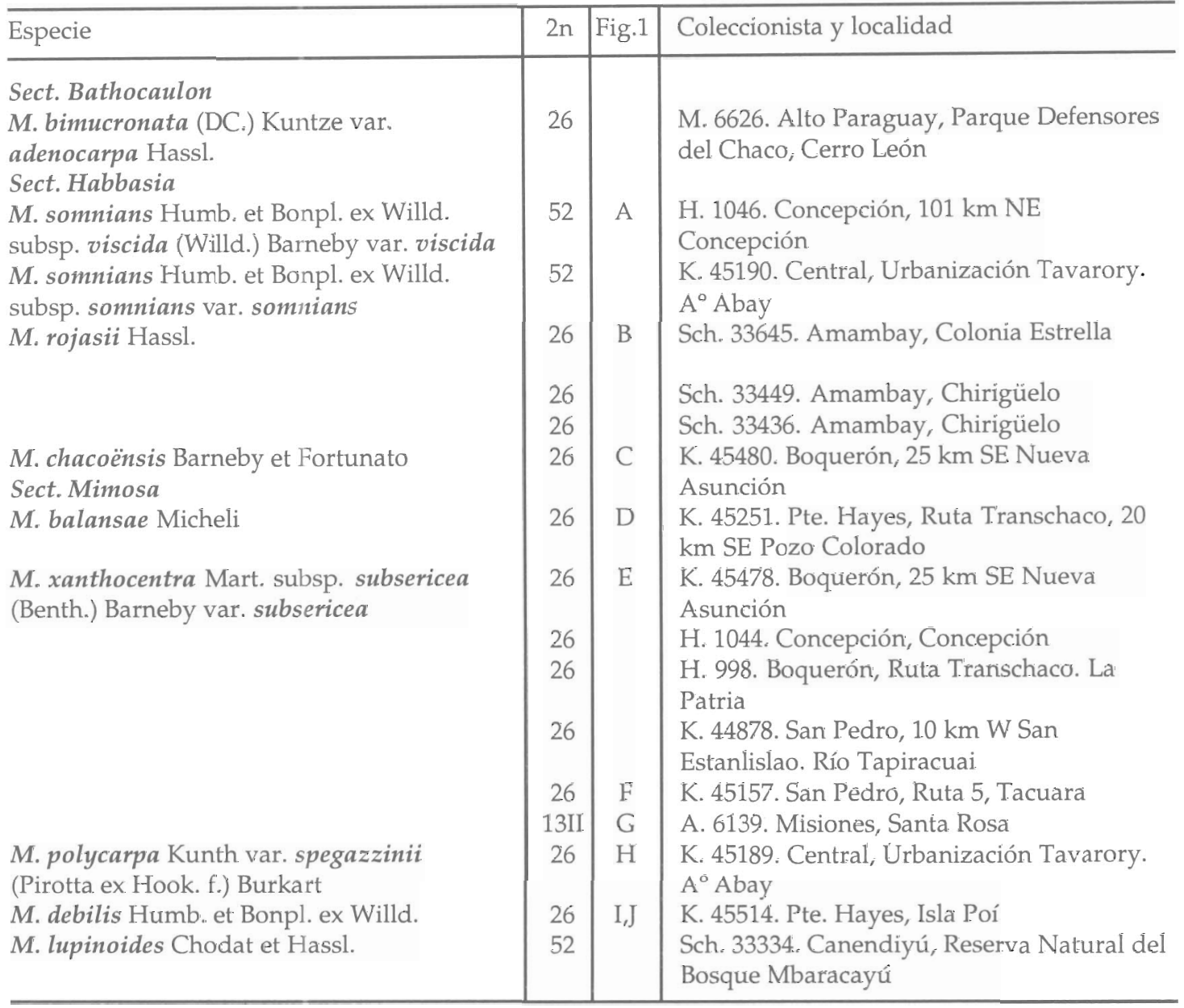

$\mathrm{A} .=$ Arbo, $\mathrm{M} \cdot \mathrm{M}$.

Sch. $=$ Schinini, A. y M. Dematteis

$\mathrm{K} .=$ Krapovickas, A.
H. $=$ Hacker, J. B. M. $=$ Mereles, $\mathrm{F}$.
Los números cromosómicos de M. xanthocentra Mart. subsp. subsericea (Benth.) Barneby var. subsericea, M. balansae Micheli, M. chacoënsis Barneby et Fortunato, $M$. rojasii Hassl. todos con $2 \mathrm{n}=26$ y de $M$. lupinoides Chodat et Hassl. con $2 n=52$ son nuevos recuentos. Las seis accesiones de $M$. xanthocentra subsp. subsericea var. subsericea presentan el mismo número diploide $2 n=26$.

Las dos accesiones de M. somnians Humb. et Bonpl. ex Willd. estudiadas aquí presentan $2 \mathrm{n}=52$, siendo por tanto tetraploides. Cada accesión pertenece a una subespecie distinta, subsp. somnians y subsp. viscida (Willd.) Barneby, las que difieren en distribución y caracteres morfológicos. La subsp. somnians se distribuye desde México hasta Argentina, mientras la subsp. viscida vive en el centro-este de Sudamérica; además, se diferencian por la presencia de acúleos y tipo de indumento. El número cromosómico para el ejemplar de la subsp. viscida var. viscida es novedad, mientras que para la subsp. somnians var. somnians ya se conocía para una población de Argentina. Sin embargo, la accesión de M. somnians var. somnians de Paraguay es tetraploide, mientras 


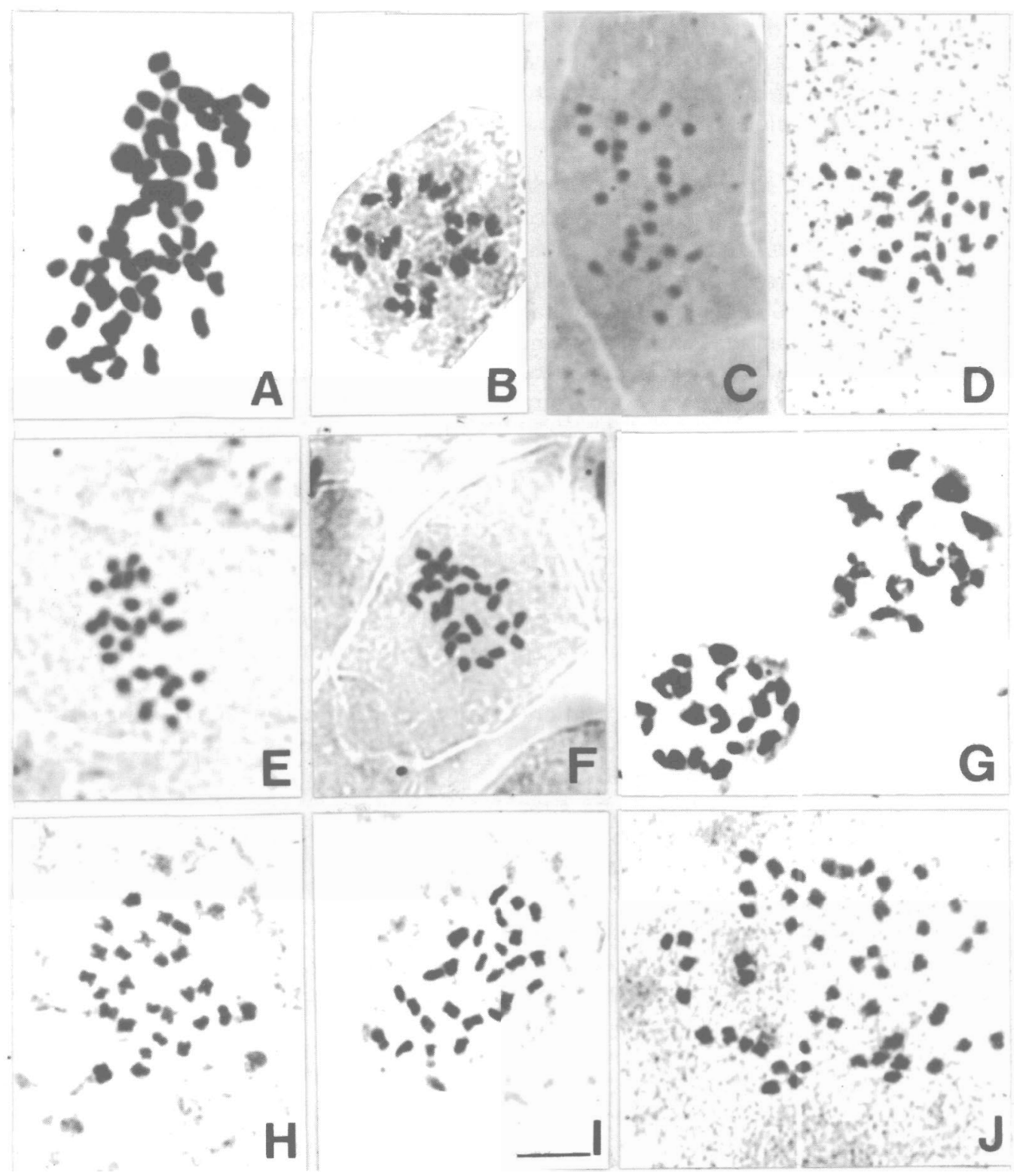

Fig. 1. A, M. somnians subsp. viscida var. viscida $2 n=52 ; B, M$. rojasii $2 n=26 ; C, M$. chacoënsis $2 n=26 ; D, M$. balansae $2 n=26$. $E$ G, M. xanthocentra subsp. subsericea var. subsericea. $\mathrm{E}$, mitosis (K.45478) $2 n=26$; $F$, mitosis (K. 45157) 2n=26; G, meiosis (A. 6139) $2 \mathrm{n}=13 \mathrm{II} ; \mathrm{H}, M$. poly carpa var.spegazzinii $2 \mathrm{n}=26 ; \mathrm{I}$; $M$. debilis, célula diploide $2 \mathrm{n}=2 \mathrm{x}=26 ; \mathrm{J}$, M. debilis, célula polisomática con $2 n=4 x=52$. La barra indica $5 \mu$ m.

que la accesión argentina de la misma variedad estudiada previamente es diploide, con $2 n=26$ (Seijo, 1993).

M. debilis Humb. et Bonpl. ex Willd. presentó $2 \mathrm{n}=26$, número que difiere del publicado para las accesiones argentinas de $M$. debilis var. debilis, que son tetraploides, con $2 n=52$ (Seijo,
1993, 1999). El ejemplar estudiado difiere de las variedades típica y parapitiensis por ser de porte más robusto y poseer tallos con muy escasos pelos y acúleos; y también de la variedad vestita, ya que esta última es completamente inerme, por lo que no es posible asignarlo con certeza a ninguna variedad. 
La existencia de individuos diploides y tetraploides dentro de la misma especie, señala la necesidad de intensificar los estudios tanto citogenéticos como taxonómicos en $M$. somnians y en $M$. debilis.

M. bimucronata (DC.) Kuntze var. adenocarpa Hassl. presenta el mismo número cromosómico $2 n=26$ que las accesiones argentinas de la misma especie pertenecientes a la var. bimucronata (Covas \& Schnack, 1947; Seijo, 1999). Ambas variedades se diferencian por la fórmula foliar, el tamaño de los folíolos y la distribución geográfica. La variedad adenocarpa se conoce para el noreste de Paraguay, mientras que la variedad bimucronata se extiende por la cuenca de los ríos Paraguay y Paraná, llegando hasta la costa Atlántica.

Por último, la accesión de M. polycarpa Kunth var. spegazzinii (Pirotta ex Hook. f.) Burkart tiene cl mismo número cromosómico que ejemplares argentinos de la misma variedad, $2 n=26$ (Seijo, 1993, 1999).

En todas las especies estudiadas se han observado células polisomáticas con números cromosómicos duplicados y cuadruplicados, o sea con $4 \mathrm{x}$ (Fig. 1J) y $8 \mathrm{x}$, como ya ha sido descripto para otras especies de Mimosa (Witkus \& Berger, 1947; Covas \& Schnack, 1947; Seijo, 1993).

Los números cromosómicos de todas las especies estudiadas en este trabajo son múltiplos del número básico $x=13$. Considerando los números cromosómicos publicados para Mimosa (Darlington \& Wylie, 1956; Fedorov, 1974; Moore, 1973, 1977; Goldblatt, 1981a, 1984, 1985, 1988; Goldblatt \& Johnson, 1990, 1991), varios números básicos pueden ser supuestos; sin embargo, los datos de este trabajo y anteriores (Seijo, 1993, 1999) indican que el número básico del género sería $x=13$. Goldblatt (1981b) propone $x=13$ como único número básico para el género y sugiere que los otros números básicos corresponderían a recuentos cromosómicos erróneos. Este hecho podría deberse al pequeño tamaño de los cromosomas y a las dificultades técnicas que presenta el material para obtener buena dispersión de los cromosomas en metafase.

De los 10 taxones estudiados aquí dos son tetraploides, con $2 n=52$, y los restantes son diploides, con 2n=26. La poliploidía es el cam- bio cromosómico más frecuentemente detectado en el género, habiéndose encontrado especies diploides, tetraploides y octoploides. El número cromosómico más común es el diploide $2 n=26$, seguido por los tetraploides y luego por los octoploides. No se han encontrado especies hexaploides ni poliploides impares, lo que sugiere que los cambios en el nivel de ploidía se dan en forma de $2^{\mathrm{n}}$. Los números cromosómicos encontrados hasta el momento se corresponden con la filogenia propuesta por Barneby (1991) ya que la mayoría de las especies poliploides como las que presentan más de un citotipo pertenecen a las secciones que este autor propone como más evolucionadas.

La poliploidía es considerada como el modo de especiación cromosómica más importante en las Angiospermas (Grant, 1971; Stebbins, 1971), a tal punto que el $30-35 \%$ de las mismas son poliploides. Mimosa no es la excepción, siendo la poliploidía uno de los factores más importantes en la especiación del género, ya que aproximadamente el 30\% de las especies estudiadas hasta ahora son poliploides.

\section{Bibliografía}

Barneby, E. 1991. Sensitivae Censitae. A description of the genus Mimosa Linnaeus (Mimosaceue) in the New World. Mem. New York Bot. Gard. 65 : 1-835.

Covas, G. \& B. Schnack, 1947. Estudios cariológicos en Antófitas. II parte. Revista Argent. Agron. 14 : 224231

Darlington, C. D. \& A. P. Wylie, (eds.) 1956. Chromosome atlas of flowering plants. MacMillan Company. New York.

Fedorov, A., ed. 1974. Chromosome numbers in flowering plants, pp. 1-928. O. Koeltz. Sci. Publ., Koenigstein.

Goldblatt, P. (ed.) 1981a. Index to plant chromosome numbers. 1975-1978. Monogr. Syst. Bot. Missouri Bot. Gard. 5.

— 1981b. Cytology and the phylogeny of Leguminosae. In: R. M. Polhill and P. H. Raven (eds.), Advances in legume systematics, Part 2: 427-464. Royal Botanic Gardens, Kew.

_ 1984. Index to plant chromosome numbers. 1979-1981. Monogr. Syst. Bot. Missouri Bot. Gard. 8.

— 1985. Index to plant chromosome numbers. 1982-1983. Monogr. Syst. Bot. Missouri Bot. Gard. 13.

— 1988. Index to plant chromosome numbers. 1984-1985. Monogr. Syst. Bot. Missouri Bot. Gard. 23.

Goldblatt, P. \& D. E. Johnson, (eds.) 1990. Index to plant chromosome numbers. 1986-1987. Monogr. Syst. Bot. Missouri Bot. Gard. 23.

1991. Index to plant chromosome numbers. 1988-1989. 
Monogr. Syst. Bot. Missouri Bot. Gard. 40.

Grant, V. 1971. Plantspeciation. Columbia University Press. Moore, R. E., (ed.) 1973. Index to chromosome numbers for 1967-1971. Regnum Veg. 90 : 1-539.

- 1977. Index to plant chromosome numbers for 19731974. Regnum Veg. $96: 1-257$.

Seijo, G. 1993. Números cromosómicos en especies argentinas del género Mimosa (Leguminosae). Bol. Soc. Argent.
Bot. 29 : 219-223

- 1999. Chromosome studies in argentinian species of Mimosa. Cytologia 64: 241-246.

Stebbins, G. L. 1971. Chromosome evolution in higher plants. Edward Amold (publishers) Ltd.

Witkus, E. R. \& C. A. Berger 1947. Polyploid mitosis in the normal development of Mimosa pudica. Bull. Torrey Bot. Club $74: 279-282$. 